\title{
Historische Soziologie
}

\author{
Peter Fischer
}

Angenommen: 7. Dezember 2021 / Online publiziert: 27. Januar 2022

(C) Der/die Autor(en) 2022

Lachmann, Richard: First-Class Passengers on a Sinking Ship. Elite Politics and the Decline of Great Powers. London, New York: Verso 2020. 477 Seiten. ISBN 978178873-407-3. Preis: \$34,95.

Der Titel des jüngsten Buches des Soziologen aus Albany (New York, USA) weckt filmische Assoziationen und provoziert die Frage, ob Passagiere aus der ersten Klasse auf einem sinkenden Schiff bevorzugt Zugang zu den Rettungsbooten erhalten. Die Antwort aus Perspektive einer Elitesoziologie kann wenig überraschen: ja. Folgerichtig steht der Titel als Metapher für Lachmanns Prognose des ökonomischen, militärischen und demokratischen Niedergangs der USA. Lachmann ist Vertreter der Historischen Soziologie, einem Paradigma, welches in Deutschland gelegentlich noch unter dem Verdacht steht, Geschichtsphilosophie oder bestenfalls Sozialgeschichte zu sein. Beide Verdachtsmomente können mit Blick auf das vorliegende Werk entkräftet werden. Der Verfasser stellt sich die Aufgabe, das Problem des Niedergangs von Imperien historisch-komparativ und in Bezug auf die aktuelle Situation der USA aufzuarbeiten. Vereinfacht gesagt ist das Erkenntnisinteresse der Historischen Soziologie die Erklärung des historischen Wandels oder des historischen Gewordenseins der Gegenwartsgesellschaft durch die Aufdeckung von Mustern, Strukturen und Entwicklungspfaden - so auch im vorliegenden Fall. George Steinmetz hat diese Grundidee der Historischen Soziologie auf den Punkt gebracht, wenn er schreibt: „Geschichte wiederholt sich nicht, aber sie reimt sich.“ Es ist nicht möglich, ausführlicher auf Programmatik und Spielarten der Historischen Soziolo-

P. Fischer $(\bowtie)$

Institut für Soziologie, Technische Universität Dresden

Helmholtzstr. 10, 01069 Dresden, Deutschland

E-Mail: peter.fischer1@tu-dresden.de 
gie einzugehen, es sei aber auf Richard Lachmanns Einführung What is Historical Sociology aus dem Jahr 2013 hingewiesen.

Wie der Untertitel andeutet, ist das Thema des Buches die Bedeutung der Eliten beim Aufstieg und Fall von Imperien. Denn, so die These des Verfassers, der Niedergang von Großmächten ist ursächlich mit Elitekonflikten verbunden, ,contingent chains of elite conflict and structural change determined the viability of modern empires, their relative geopolitical and economic standing, whether they became hegemons and how long and in what form their hegemony endured" (S. 13). Im Detail bietet Lachmanns quellengesättigte und luzide Studie aber mehr als eine Elitesoziologie, sie erörtert Themen, die in den letzten Jahren ein verstärktes Interesse innerhalb der Historischen Soziologie erfahren haben: so z. B. eine Soziologie des Imperiums, die nach Entstehung und Zerfall von hegemonialer Macht fragt, eine Diskussion um die Weiterentwicklung der Weltsystemtheorie von Wallerstein, aber auch die Bewertung der Bedeutung von Kolonien bei der Entstehung von Staaten und Imperien.

Lachmann macht sechs Imperien in der kapitalistischen Ära aus, die über ausreichend Macht verfügen, um als militärisch, ökonomisch oder geopolitisch dominant zu gelten: Spanien, Frankreich, Portugal, die Niederlande, Großbritannien und die USA (vgl. S. 25). Doch Dominanz oder Hegemonie in einem oder mehreren Bereichen bedeutet nicht gleich ein Imperium. Dem Autor folgend kann nur vier von diesen sechs der Status zugesprochen werden. Spanien und Frankreich verfügten zwar über militärische Macht, waren aber nicht in der Lage, diese in ein kohärentes Imperium zu überführen. Das Osmanische Reich, Österreich-Ungarn und Russland scheiden als Kandidaten für ein Imperium aus, denn keiner von diesen drei ,were able, or even sought, to parlay their collection of dependencies into military power beyond Eurasia or into any form of economic dominance“ (S. 25). Richtig ist: im Laufe der Geschichte gab es zahlreiche Imperien, von denen aber nur wenige die Hegemonie besaßen, um weltweite Spielregeln auch für andere mächtige Länder aufzustellen. Lachmann fragt daher ,how one policy achieved and held leverage that allowed it to shape the operations of the world capitalist system and geopolitical order to its singular advantage" (S. 49). Der Weg zu einem kohärenten Imperium ist von mehreren Faktoren abhängig, zu nennen sind z. B. die Herausforderung durch rivalisierende geopolitische Kräfte, das Ausmaß des kapitalistischem Wettbewerbs, das Streben nach Eigennutz von Eliten in der Metropole oder aber der Widerstand untergeordneter oder kolonialer Eliten gegenüber der zentralen Herrschaft. Mit Blick auf diese konstituierenden Faktoren wird für Lachmann ersichtlich, dass vor allem Eliten am Werke sind: „Elites make empires, and empires create new elites and change the structure of relations among old and new elites“ (S. 26). Das Argument klingt simpel, gewinnt aber im Verlauf der Analyse zunehmend an Komplexität, denn die Typisierung des Empires erfolgt durch seine jeweilige Elitenstruktur (vgl. S. 31, S. 54), was sich z.B. in einer Kreuztabelle darstellen lässt, in der die Imperien bezüglich Merkmalen befragt werden. Gibt es Elitenkonflikte? Ist die Autonomie der Eliten in den Kolonien zu hoch? Ist die Einheit der Elite, die über andere Eliten herrscht, gesichert? Sind infrastrukturelle Möglichkeiten ausgeprägt, um untergeordnete Eliten zu kontrollieren? Jedes dieser Merkmale könne, so die These, die Herausbildung eines hegemonialen Imperiums verhindern. Um ein Bei- 
spiel aufzugreifen: Die Bedeutung von Kolonien für die Bildung eines Imperiums ist offensichtlich, reicht aber kaum hin, um ein solches auf Dauer zu stellen. Zu hohe Autonomie der Eliten in den Kolonien ist hinderlich für die Einheit des Reichs, ein zu hohes Maß an Elitenkonsens kann aber auf Dauer ebenso problematisch sein, wie das Beispiel Großbritanniens zeigt: „Rather consenus among interlocking elites allowed an enduring imperial and fiscal policy that actually was able to serve all those elites' self perceived interests but that blocked reforms, vital to countering the rise of economic and military rivals" (S. 240).

Einen Großteil des Buches nimmt die Darstellung von „Hegemony Past“ und „Hegemony Present“ ein. Für die Vergangenheit schildert Lachmann den Auf- und Abstieg der Niederlande und Großbritanniens. Der Verfasser argumentiert detailliert, sprachlich präzise und inhaltlich komplex, sodass bereits die auszugsweise Lektüre gewinnbringend ist. Die hegemoniale Gegenwart beginnt ihm zufolge mit dem Aufstieg der USA seit den 1960er-Jahren, allerdings sei der anfängliche Elitekonsens mittlerweile einer Paralyse gewichen (vgl. S. 243). Sowohl Militär als auch Ökonomie und politische Steuerung der USA befänden sich im Blick über „longue durée“ im Niedergang, einzig der Bereich der Finanzialisierung stehe noch unter amerikanischer Vorherrschaft. Für den Verfall nennt Lachmann fünf Gründe, die ebenfalls luzid und quellengesättigt aufgearbeitet werden: den ökonomischen Niedergang seit den 1970er-Jahren, den Verlust geopolitischer Hegemonie, das Ende des Kalten Krieges, die gesellschaftliche Mobilisierung von links und schließlich die Mobilisierung von rechts. Lachmanns Beweisführung ist schlüssig, so z. B. seine Analyse zum Niedergang des amerikanischen Militärs, die gleichzeitig ernüchterndes und erhellendes zutage bringt, wenn er die Armee als „,collection of tribes“ schildert (vgl. S. 324). Das Militär bleibe zwar letztlich ohne wirklichen Gegner, aber auch seit langer Zeit ohne Sieg, sehe man vom ersten Golfkrieg und einigen kleineren Eingriffen ab. In der Gesamttendenz konstatiert der Verfasser den USA das gleiche Schicksal wie Großbritannien und den Niederlanden, einen irreversiblen Niedergang. Das wirft Fragen auf, z.B. was nach dem Verlust der Hegemonialstellung kommt. Nationaler Aufschwung oder Abstieg, beides ist denkbar. Lachmann sieht in China nicht den Nachfolger der USA als ökonomischer Weltmacht, trotz dessen steigender Macht in Eurasien. Auch militärisch seien starke Weltmächte Verbündete der USA, sodass weder China noch Russland ernsthaftes Interesse daran hätten, die USA herauszufordern. Wie andere Imperien wird Amerika nach dem Niedergang nicht von der Bildfläche verschwinden, seine Bedeutung aber allmählich abnehmen. Der Verfall hinterlässt aber Spuren, nicht zuletzt für die Demokratie. Lachmann erwartet, dass die Vereinigten Staaten und seine Eliten zunehmend vor Angst getrieben soziale Strukturen oligarchischer werden lassen. Am Ende des Buches heißt es: „Elites to the life boat. At home or abroad, those who profited from the era of American Hegenomy and from its decline will be able to insulate themselves from their power and greed“ (S. 462). Die Möglichkeit, dass sie aber doch zusammen mit allen anderen untergehen, bleibt bestehen.

Ohne Zweifel, Lachmanns Buch ist beeindruckend und besticht vor allem durch seine analytische Stärke, die häufig an Max Weber erinnert. Die Elitesoziologie, lange Zeit aus dem Mainstream des Fachs verdrängt und seit einigen Jahren wieder verstärkt im Fokus, erhält hier eine wichtige historische Wendung. Dennoch 
liefert Lachmann keine Elitentheorie, er gibt auch kaum eine präzise Bestimmung von Eliten und ihrer Beziehung zueinander sowie keine theoretische Systematisierung des Konflikts. Er folgt der Machtheorie von Mann, denn an der Spitze von Machtstrukturen stehen Eliten. Das ist natürlich alles richtig, aber andere Eliten, wie z.B. Medienvertreter oder ,public intellectuals“, tauchen kaum auf. Es bleiben daher offene Fragen, die zumindest teilweise in den USA seit Erscheinen des Buchs diskutiert werden (vgl. das Symposium zum Buch im International Journal of Comparative Sociology 6/21) Neben dem Streit über die Anzahl von hegemonialen Imperien scheint die Frage nach der Bedeutung von Eliten im Gegensatz zu anderen Aspekten wie Strukturen oder geografischen Räumen für die Herausbildung von hegemonialen Imperien wichtig. Auffallend ist aber auch, dass Lachmann zwar analytisch dicht, theoretisch aber eher defensiv argumentiert. Eine theoretische $\mathrm{Zu}-$ spitzung der aus der komparativen Analyse von Großbritannien, den Niederlanden und den USA gewonnen Erkenntnisse wäre so vielleicht hilfreicher gewesen als Empfehlungen und Hoffnungen, wie es nach dem Niedergang weitergehen könnte. Das Buch bleibt dennoch absolut empfehlenswert.

Funding Open Access funding enabled and organized by Projekt DEAL.

Open Access Dieser Artikel wird unter der Creative Commons Namensnennung 4.0 International Lizenz veröffentlicht, welche die Nutzung, Vervielfältigung, Bearbeitung, Verbreitung und Wiedergabe in jeglichem Medium und Format erlaubt, sofern Sie den/die ursprünglichen Autor(en) und die Quelle ordnungsgemäß nennen, einen Link zur Creative Commons Lizenz beifügen und angeben, ob Änderungen vorgenommen wurden.

Die in diesem Artikel enthaltenen Bilder und sonstiges Drittmaterial unterliegen ebenfalls der genannten Creative Commons Lizenz, sofern sich aus der Abbildungslegende nichts anderes ergibt. Sofern das betreffende Material nicht unter der genannten Creative Commons Lizenz steht und die betreffende Handlung nicht nach gesetzlichen Vorschriften erlaubt ist, ist für die oben aufgeführten Weiterverwendungen des Materials die Einwilligung des jeweiligen Rechteinhabers einzuholen.

Weitere Details zur Lizenz entnehmen Sie bitte der Lizenzinformation auf http://creativecommons.org/ licenses/by/4.0/deed.de.

Peter Fischer Dr. phil., ist Soziologe und wurde am 15.09.2021 zur Habilitation an der Philosophischen Fakultät der TU Dresden zugelassen. Forschungsschwerpunkte: Historische Soziologie, Wissenssoziologie, Allgemeine Soziologie. Aktuelle Publikationen: Gesellschaftliche Krise, Neokonservatismus und die „neuen“ pädagogischen Ideen. In: Das Ende der politischen Ordnungsvorstellungen des 20. Jahrhunderts, Hrsg. U. Binder und J. Oelkers. Wiesbaden 2020; Die Emigration deutscher Soziologie in die USA als Motor der historischen Soziologie. Das Beispiel Reinhard Bendix. In: Gesellschaftliche Spannungen als historische Motoren der Soziologie, Hrsg. N. Holzhauser, A. Ploder und S. Moebius. Wiesbaden (im Erscheinen). 